\title{
Multiple paths to the Kuiper belt
}

J. Spacecr. Rockets (in the press); preprint available

at https://arxiv.org/abs/1810.07811

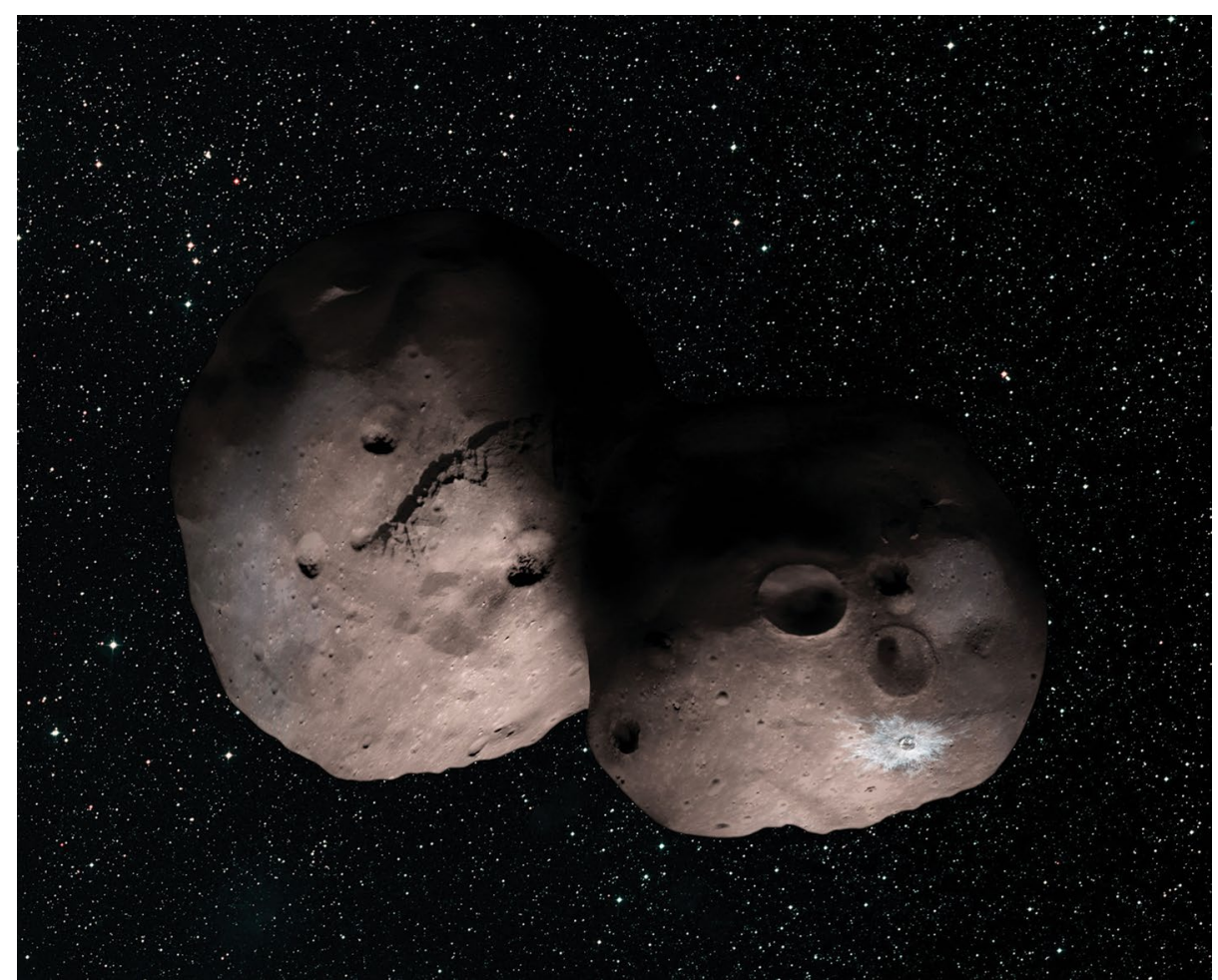

Credit: NASA/Johns Hopkins University Applied Physics Laboratory/Southwest Research Institute/ Alex Parker

After the successful trip to Pluto and its moon Charon, New Horizons will next rendezvous with Kuiper belt object (KBO) 2014 MU69 on 1 January 2019. It will be the most distant flyby yet, and given the odd shape of MU69 (one possible form is pictured), the excitement is palpable. However, Amanda Zangari and colleagues already have their sights on the 2025-2040 launch window for a future mission back to the Kuiper belt.

Given that NASA budgets are limited, and there may be some uncertainty come January with the exit of several US Representatives with strong interest in space (among them Europa-enthusiast John Culberson), future missions must be as efficient and ambitious as possible. Rather than focus on a dedicated trip to a specific object, the authors explore a number of trajectories that can fly by various outer planets and $46 \mathrm{KBOs}$, including Pluto and all named KBOs of magnitude $H>4$, within a span of 25 years.

Using the Mako code, they find that the most efficient trajectory to visit all $46 \mathrm{KBOs}$, in terms of launch energy and total travel time, would be a direct gravity assist by Jupiter. Adding another giant planet in the swingby would only have the effect of reducing the number of reachable KBOs within the same timeframe. Saturnbased trajectories to the KBOs are also considered, but have a later launch window than a Jupiter flyby. The science return of the various options will have to be carefully assessed in order to put together a strong proposal for the next Planetary Decadal survey.

\section{May Chiao}

Published online: 23 November 2018

https://doi.org/10.1038/s41550-018-0652-4 